\title{
The Dor procedure: What has changed after fifteen years of clinical practice?
}

\section{Lorenzo Menicanti, MDa \\ Marisa Di Donato, $\mathrm{MD}^{\mathrm{b}}$}

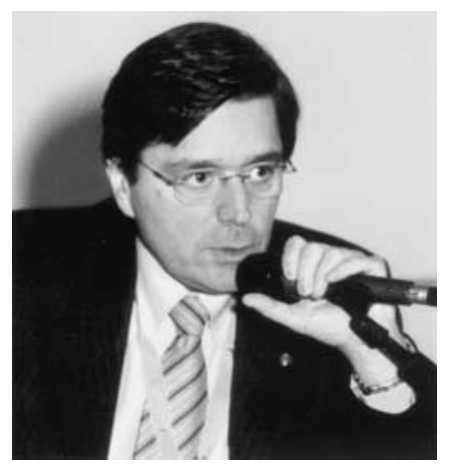

Dr Menicanti

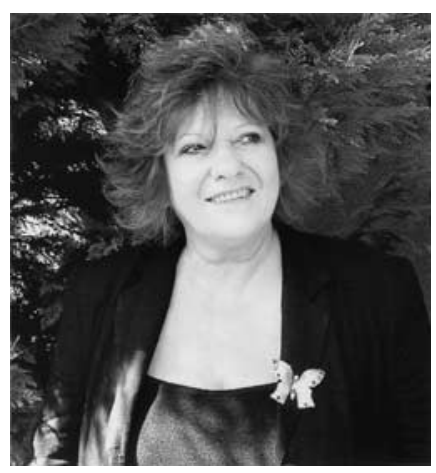

Dr Di Donato

See related article on page 863 .

From San Donato Hospital, Milan, Italy, ${ }^{a}$ and the Department of Critical Care Medicine, University of Florence, Florence, Italy. ${ }^{\mathrm{b}}$

Received for publication June 27, 2002; accepted for publication July 1, 2002.

Address for reprints: Lorenzo Menicanti, MD, Chief in Cardiac Surgery, Istituto Policlinico San Donato, Cardiac Surgery, Via Morandi 30, San Donato Milanese 20097, Italy.

J Thorac Cardiovasc Surg 2002;124:886-90

Copyright () 2002 by The American Association for Thoracic Surgery

0022-5223/2002 \$35.00+0 $\quad \mathbf{1 2 / 1 / 1 2 9 1 4 0}$

doi:10.1067/mtc.2002.129140

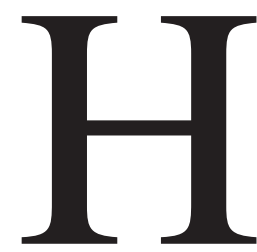

eart failure is a major health problem with increasing prevalence due partly to an aged population and more effective treatment of acute myocardial infarction. Postinfarction left ventricular remodeling is characterized by chamber dilatation and abnormal shape leading to systolic and diastolic dysfunction. In advanced form, it leads to the heart failure syndrome and is progressive. Intensive medical management reduces symptoms and improves survival. However, patients who are in functional class III or IV have a poor 3-year prognosis. ${ }^{1-3}$ Recently, surgical approaches have been designed to abort and reverse remodeling, diminish heart failure, and improve survival. With minor modifications, this surgical approach uses the Dor procedure.

Surgical therapy achieves the following:

1. Relieves ischemia by coronary revascularization.

2. Diminishes ventricular volume.

3. Restores the ventricle to more normal geometry.

4. Further diminishes volume overload by mitral valve repair when appropriate.

\section{History of the Dor Procedure}

In 1985, Dor described an original surgical technique built on prior contributions by Cooley, Keith, and Jatene. ${ }^{4-7}$ The Dor procedure excludes akinetic or dyskinetic portions of the ventricle, reshapes the ventricle with a stitch that encircles the transitional zone between contractile and noncontractile myocardium, and uses a small patch to reestablish ventricular wall continuity at the level of the purse-string suture.

\section{Pathophysiology of Postinfarction Remodeling}

Early reperfusion procedures for acute myocardial infarction, whether by thrombolysis or angioplasty, have altered the pathologic changes that follow acute myocardial infarction. Reperfusion produces epicardial and, occasionally, midmyocardial sparing while leaving endocardial necrosis. Persistence of viable ventricular muscle frequently trades left ventricular dyssynergy for asynergy, with some preservation of wall thickness. When one third or more of the ventricular perimeter is involved, left ventricular volume markedly increases, the apical and basal portions become rounded, and pump function is globally depressed. This condition resembles dilated nonischemic cardiomyopathy more than classic dyskinetic aneurysm. Extension of modified aneurysm surgery to large dilated ventricles has been made possible by the Dor procedure. ${ }^{89}$ Di Donato has demonstrated that outcome in a large series of surgically treated patients is more strongly linked to the extent of asynergy than to the type of asynergy (akinetic vs dyskinetic). ${ }^{10}$ This observation has helped advance the belief that remodeling operations may be applied to the surgical treatment of postinfarction dilated cardiomyopathy.

\section{Pathophysiologic Basis for the Components of the Dor Procedure Relieve Ischemia}

Coronary revascularization should be as complete as possible. Grafting the left anterior descending coronary artery (LAD) is important since the high portion of the 


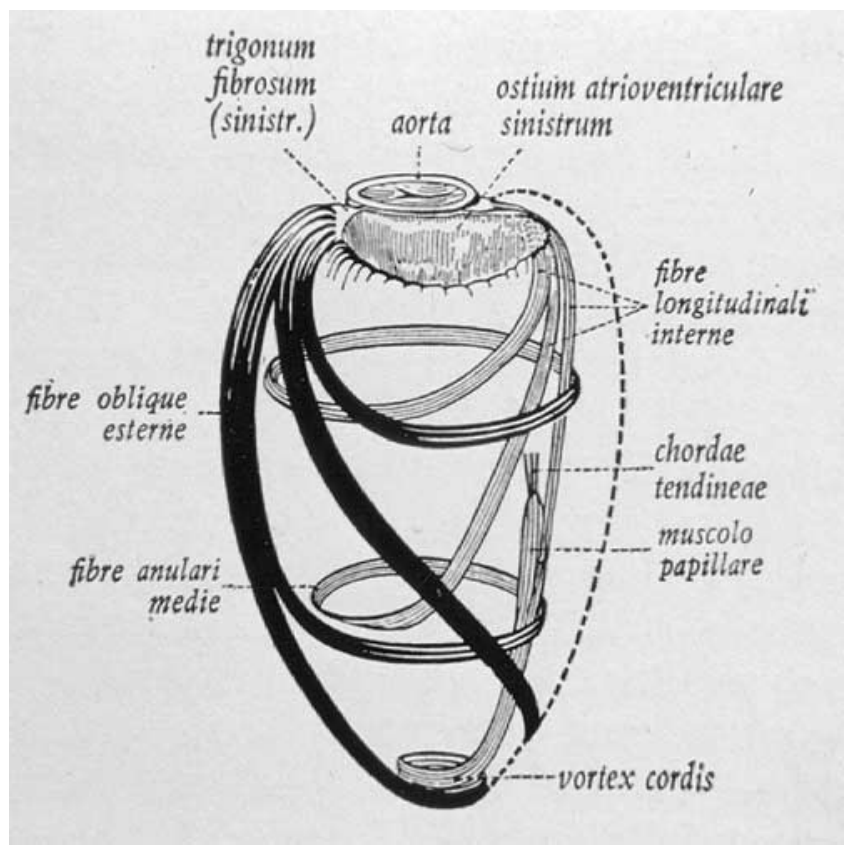

Figure 1. Drawing showing that the myocardial fibers of the normal heart have a spiral direction from the base to the apex with two opposite layers and well-defined intersecting angles. (From Benninghoff-Goertler, Atlas of anatomy, Vol II, 1996, Piccin Editor. Published with permission).

septum, which is almost always functioning, needs to be perfused. In our early experience 70 patients underwent pre-coronary and post-coronary angiography. All patients had a critically stenosed LAD, and in 35 of them the LAD, distal to the lesion, was not visible at angiography. All had left internal thoracic artery grafts and 27 of 35 patients had a patent graft with a 3-month patency of $77 \% .{ }^{11}$

\section{Diminish Ventricular Volume}

Ventricular volume should be reduced in its septal and anterior components without deforming the chamber. If the residual volume is too small, the results will be catastrophic, resulting in the physiology of a restrictive cardiomyopathy. This risk is particularly great if the preoperative chamber is only moderately dilated. If the residual chamber is too large, the benefit will be limited. To diminish this risk, Dor introduced the use of an intraventricular balloon filled to a known volume of $60 \mathrm{~mL} / \mathrm{m}^{2}$, to guide the restoration and to leave an adequate residual chamber. The volume $60 \mathrm{~mL} / \mathrm{m}^{2}$ was chosen after study of postoperative angiograms. This value may be too small if the preoperative volume is very large; thus, when we approach preoperative volumes greater than $150 \mathrm{~mL} / \mathrm{m}^{2}$, we add $15 \%$ to the volume of the balloon (approximately $70 \mathrm{~mL} / \mathrm{m}^{2}$ ).

The opening of the ventricle is closed with a Dacron patch if the diameter is $3 \mathrm{~cm}$ or greater. If it is smaller than
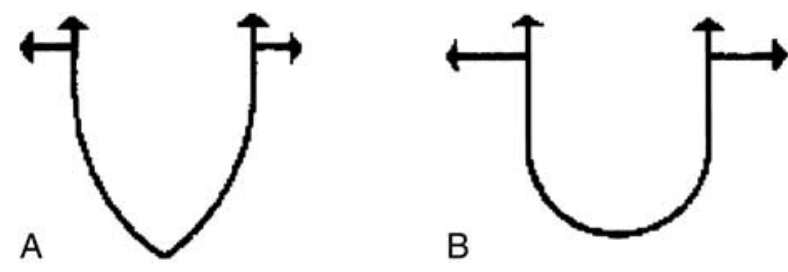

Figure 2. The Gothic $(A)$ and Roman $(B)$ arches. Arrows indicate longitudinal and lateral forces. See text for explanation.

$3 \mathrm{~cm}$ the closure is performed with simple stitches tangential to the balloon. In this case a second stratum with the excluded tissue is sutured on the first suture to avoid bleeding. If the closure is done by patch, few millimeters of external borders are left in the everting way so that it is easy to add stitches for good hemostasis, if needed. We think it is extremely important that the heart be arrested and relaxed during the procedure to allow a precise sizing of the chamber and easy closure of the ventriculotomy.

Reduction of ventricular volume has two important effects. First, based on the Laplace equation, which relates wall stress inversely to wall thickness and directly to chamber radius, volume reduction diminishes wall stress and thereby reduces myocardial oxygen consumption. Minimizing the mass of abnormal myocardium improves wall compliance, reduces filling pressure, and further enhances diastolic coronary flow. Second, reduction of wall stress, as a critical determinant of afterload, enhances contractile performance of the ventricle by increasing the extent and velocity of systolic fiber shortening. ${ }^{12}$

\section{Restore Shape}

The Dor procedure was initially perceived as functional amputation of the ventricle with exclusion of the entire akinetic or dyskinetic scar. This led to increased sphericity of the ventricle in some patients, but in general the volume reduction still improved function. However, a suboptimal short axis/long axis ratio may influence the development of late moderate mitral regurgitation. ${ }^{13}$

The objective in optimizing the shape of the ventricle should be the proportional reduction of both the short and the long axes. There are limits to the extent to which the short axis can be reduced. Circumferential shortening is maintained by the upper part of the septum and by the inferior or lateral wall, which are often sound. Their motion is crucial for a good outcome. It is therefore necessary to find equilibrium between the exclusion of akinetic or dyskinetic regions and the reduction of the longitudinal axis that is determined by the position of the new apex. To overcome this problem, we pay great attention to positioning the patch with an oblique orientation, toward the aortic outflow tract. In this way we avoid creating a boxlike shape of the ventricle that may occur when the orientation of the patch is almost parallel to the mitral valve. 


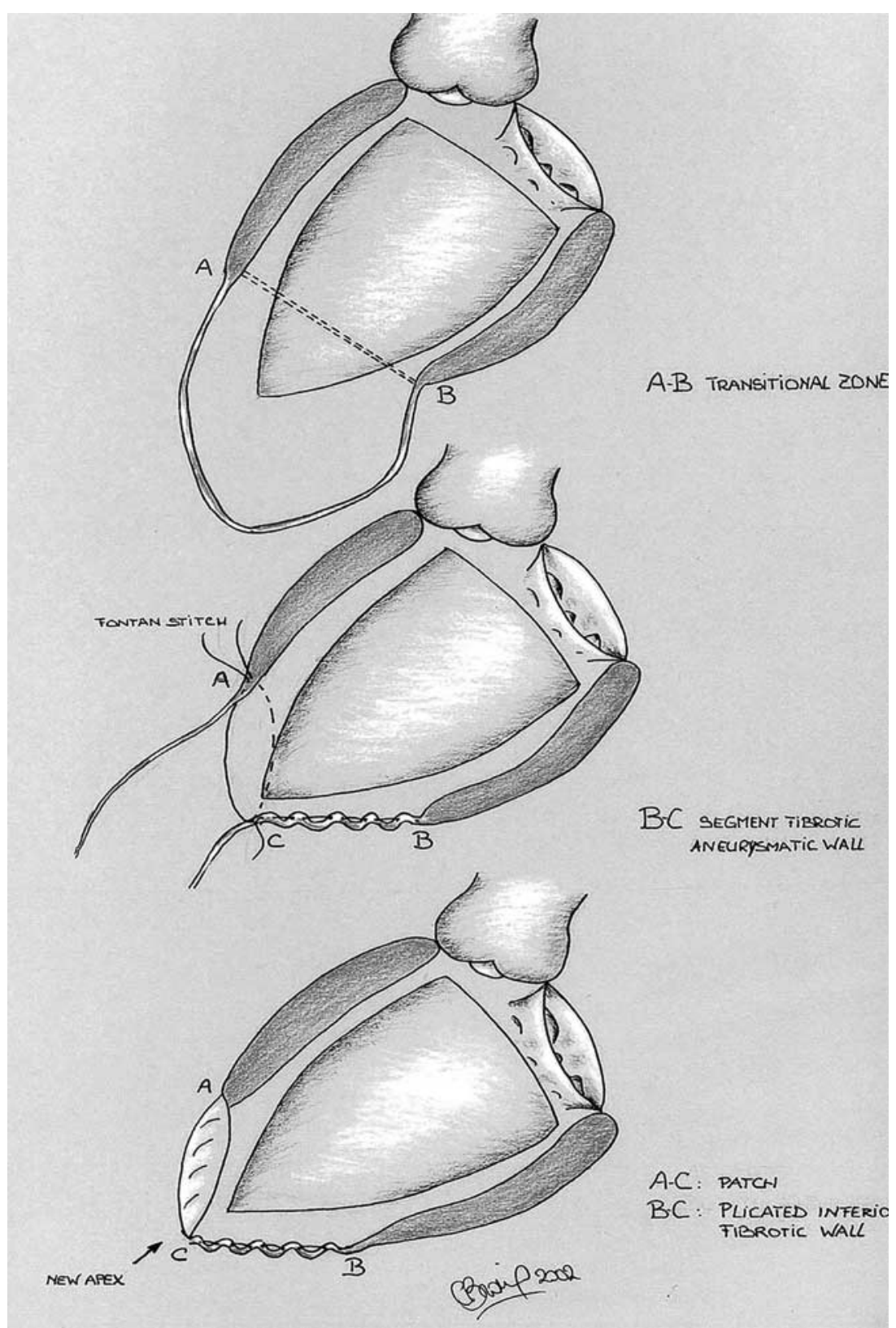

Figure 3. Reconfiguration of the ventricle. See text for explanation.

In a normal heart, myocardial fibers have a spiral direction from the base to the apex with two opposite layers and well-defined intersecting angles (Figure 1; from Benninghoff-Goertler, Vol II). This double spiral allows 30\% fiber shortening to yield a $60 \%$ ejection fraction. When the heart dilates and this spiral architecture is lost (especially at the apex), ventricular function is impaired and ejection fraction and stroke volume decrease. This starts a vicious circle, with dilatation of the ventricle and activation of peripheral and central neurohumoral mechanisms that characterize left ventricular remodeling and lead to clinical heart failure.
If we compare two different architectural arches, one with an acute and the other with a spherical form (ie, the Gothic and the Roman arch; Figure 2, $A$ and $B$, respectively) and we apply an identical force at the vertex, this force is split into two components: one longitudinal and one lateral force. For a given longitudinal component the lateral force will be higher in the spherical arch; thus, if we consider the apex of the heart as analogous to the vertex of the arch, a rounded, spherical apex will have a higher lateral force (stress) than an elliptical ventricle.

The shape of the ventricle is also important for normal 
functioning of the mitral valve. Ischemic functional mitral regurgitation is more frequent in dilated, spherical than elliptical ventricles. In a spherical heart, papillary muscles are displaced toward the lateral wall, losing their normal orientation toward the apex and increasing the distance between them. In this condition the posterior leaflet of the valve is retracted, the posterior anulus is dilated, the coaptation point is lost, and the valve becomes incompetent.

More recently, preshaped elliptical balloons (Chase Medical, Dallas, Tex) have helped size and configure the ventricle, ensuring a more normal short axis/long axis ratio. More important, this balloon gives us the correct position of the new apex. It is therefore necessary, when both the apical and inferior regions are involved in the dilatation, to leave a small portion of inferior scar during reconstruction. If the inferior region is severely dilated, we plicate it (Figure 3). A suture starting from the transitional zone will plicate the residual inferior scar, thus placing the apex in a more anterior position. The circular suture to exclude the affected anterior and septal tissue starts from this new apex, and the plane of suture is more oblique with a resultant elliptical shape.

\section{Repair Mitral Regurgitation}

If mitral regurgitation is present on the preoperative echocardiographic study, we examine the valve and perform intraoperative transesophageal echocardiography to confirm the regurgitation and to assess the results after repair. The mitral valve is well visualized from the ventriculotomy, and repair is performed from the ventricle if mitral regurgitation is greater than $2+$ or if the anulus is larger than $35 \mathrm{~mm}$. The procedure has been recently described in detail. ${ }^{14}$ In brief, a double $\mathrm{U}$ stitch on a pledget is passed posteriorly from one trigone to the other, bounding the posterior component of the mitral anulus. The stitch is then snared and secured on a second pledget. A sizer is introduced in the mitral orifice to assure an adequate mitral area; it is important to avoid leaflet involvement in the suture. If the ventriculotomy is not sufficiently large, the valve is repaired from the atrium. The advantages of repairing the valve from the ventricle are that the trigones are better visualized and the procedure is faster.

\section{Surgical Technique}

Overall, our technique does not differ from that described by Dor. ${ }^{14-17}$ The procedure is conducted under total cardiac arrest with aortic clamping. Antegrade crystalloid cardioplegia is usually applied, although more recently we have used cold blood cardioplegia with no outcome differences observed between the two types of myocardial protection. Complete coronary revascularization, including placement of the internal thoracic artery to the LAD (to preserve the septal branches) is performed first. Then, the ventricle is opened, the transitional zone and papillary muscles are carefully checked, and the balloon-based repair is completed.

\section{The Milan Experience}

We have operated on 985 patients since 1989 at the San Donato Hospital, Milan, Italy. Eighty percent of these interventions were for anteroseptal myocardial infarction. The overall operative mortality rate in anterior reconstruction was $7.2 \%$. It was $4.8 \%$ when ventricular repair was associated with coronary artery bypass grafting, $15 \%$ with an associated mitral valve procedure, and $15.3 \%$ with preoperative New York Heart Association (NYHA) functional class IV. Operative mortality in patients with a preoperative ejection fraction of $30 \%$ or less was $12.3 \%$. The mean delay from myocardial infarction was $36 \pm 55$ months, but recently $36 \%$ of patients had had a myocardial infarction 3 months earlier or less and were operated on for hemodynamic instability. Our results confirm those reported by Dor and coworkers in that patients with more severe preoperative left ventricular dysfunction benefit more from the intervention. In a series of 81 patients with a preoperative ejection fraction of $30 \%$ or less, ejection fraction improved from $25 \% \pm 4 \%$ to $35 \% \pm 9 \%(P<.000)$; end-diastolic volume from $249 \pm 80 \mathrm{~mL}$ to $165 \pm 56 \mathrm{~mL}$, and endsystolic volume from $188 \pm 64 \mathrm{~mL}$ to $107 \pm 46 \mathrm{~mL}$, respectively $(P<.000)$. NYHA functional class improved from $3 \pm 1$ to $1.6 \pm 1(P<.001)$.

Factors associated with adverse outcome in our series are as follows: worse functional class; ejection fraction less than 20\%; age more than 70 years; urgent intervention; mitral procedure (especially mitral replacement); pulmonary hypertension (systolic pulmonary artery pressure $60 \mathrm{~mm} \mathrm{Hg}$ ); the number and sites of previous myocardial infarctions; and right ventricular dysfunction. Although high-grade functional class and worse hemodynamic conditions carry a higher mortality rate, surviving patients benefit most from this operation. A recent article showed an improvement in 5-year survival in patients with high-grade preoperative left ventricular dysfunction operated on with this procedure ${ }^{17}$ and an excellent survival at 5 years of patients in NYHA class III or less. We believe that earlier intervention may, if not arrest or revert, at least attenuate remodeling and its deleterious effects on symptoms and survival.

\section{Indications}

On the basis of our experience, we consider the following to be the indications for surgery. In patients of any age:

- Anteroseptal infarction and dilated left ventricle (enddiastolic volume index $>100 \mathrm{~mL} / \mathrm{m}^{2}$ ), and

- Depressed ejection fraction (even below 20\%), and

- Left ventricular regional asynergy, either dyskinesia or akinesia, greater than $35 \%$ of the ventricular perimeter, and

- Symptoms of angina, heart failure, arrhythmias, or a combination of the three, or

- Inducible ischemia on provocative tests in asymptomatic patients. 
If patients are asymptomatic and results of provocative tests are negative, we suggest monitoring them by an echocardiographic study every 6 months. If the ventricle tends to dilate and ejection fraction tends to decline, these patients should be offered the procedure. Intervention may limit the progressive deterioration in clinical status.

\section{Relative Contraindications}

- Systolic pulmonary artery pressure more than $60 \mathrm{~mm} \mathrm{Hg}$ (when not associated with severe mitral regurgitation).

- Severe right ventricular dysfunction as assessed by TAPSE (tricuspid anulus plane systolic excursion). This is a simple index of function, and when it is less than $10 \mathrm{~mm}$ the intervention is contraindicated because failure involves largely the septum and both ventricles. In this condition left ventricular repair is not effective and mortality is high.

- Regional asynergy without dilation of the ventricle (risk of too small a residual chamber!).

\section{Conclusion}

Our experience in surgical ventricular restoration for patients after posterior infarction is large and with the Dor experience reaches about 2000 cases. During the past years we have tried to improve the technique and the selection of patients. We think that, as for any innovative technique, time and experience will allow further improvement. The limitation of our study, as of that of the RESTORE group, ${ }^{18}$ is that we do not have a control group, and we look forward to the upcoming randomized STICH trial (Surgical Treatment of Ischemic Heart Failure) that will assess the best strategy for ischemic cardiomyopathy with dilated ventricles, depressed ejection fraction, and NYHA functional class II or more. The STICH results should confirm or refute our hypothesis that surgical intervention improves quality and duration of life in patients with severe postinfarction ventricular remodeling.

\section{References}

1. Califf RM, Adams KF, McKenna WJ, et al. A randomized controlled trial of epoprostenol therapy for severe congestive heart failure: The Flolan International Randomized Survival Trial (FIRST). Am Heart J. 1997; 134:44-54.
2. Greenberg B, Quinones MA, Koilpillai C, et al. Effects of long-term enalapril therapy on cardiac structure and function in patients with left ventricular dysfunction. Circulation. 1995;91:2573-81.

3. Watson RDS, Gibbs CR, Lip GY. ABC of heart failure: clinical features and complications. BMJ. 2000;320:236-9.

4. Dor V, Kreitmann P, Jourdan J, et al. Interest of physiological closure (circumferential plasty on contractile areas) of left ventricle after resection and endocardectomy for aneurysm of akinetic zone: comparison with classical technique about a series of 209 left ventricular resections [abstract]. J Cardiovasc Surg. 1985;26:73.

5. Cooley D. Ventricular endoaneurysmorrhaphy: a simplified repair for extensive postinfarction aneurysm. J Cardiac Surg. 1989;4:200-5.

6. Loop FD, Effler DB. Left ventricular aneurysm. In: Gibbon's surgery of the chest, 3rd ed. Philadelphia: WB Saunders; 1976. p. 1384.

7. Jatene AD. Left ventricular aneurysmectomy: resection or reconstruction. J Thorac Cardiovasc Surg. 1985;89:321-31.

8. Dor V, Sabatier M, Di Donato M, et al. Late hemodynamic results after left ventricular patch repair associated with coronary grafting in patients with postinfarction akinetic or dyskinetic aneurysm of the left ventricle. J Thorac Cardiovasc Surg. 1995;110:1291-301.

9. Dor V, Sabatier M, Di Donato M, et al. Efficacy of endoventricular patch plasty repair in large postinfarction akinetic scar and severe left ventricular dysfunction: comparison with a series of large dyskinetic scar. J Thorac Cardiovasc Surg. 1998;116:50-9.

10. Di Donato M, Sabatier M, Dor V, et al. Akinetic versus dyskinetic postinfarction scar: relation to surgical outcome in patients undergoing endoventricular patch plasty repair. J Am Coll Cardiol. 1997;29:1569-75.

11. Menicanti L, Frigiola A, Mazza E, et al. Use of internal mammary artery for myocardial revascularization in left ventricular aneurysmectomy. International workshop on arterial conduits for myocardial revascularization, Milan, Italy: 1994. p. A51.

12. Di Donato M, Sabatier M, Toso A, et al. Regional myocardial performance of nonischemic zones remote from anterior wall left ventricular aneurysm: effects of aneurysmectomy. Eur Heart J. 1995;16: 1285-92.

13. Di Donato M, Sabatier M, Dor V, et al. Effects of Dor procedure on left ventricular dimension and shape and geometric correlates of mitral regurgitation one year after surgery. J Thorac Cardiovasc Surg. 2001; 121:91-6.

14. Menicanti L, Di Donato M, Frigiola A, et al. Ischemic mitral regurgitation: intraventricular papillary muscle imbrication without mitral ring during left ventricular restoration. J Thorac Cardiovasc Surg. 2002;123:1041-50.

15. Dor V, Saab M, Coste P, et al. Left ventricular aneurysm: new surgical approach. Thorac Cardiovasc Surg. 1989;37:11-9.

16. Dor V. Surgery for left ventricular aneurysm. Curr Opin Cardiol. 1990;5:773-80.

17. Di Donato M, Toso A, Maioli M, et al. Intermediate survival and predictors of death after surgical ventricular restoration. Thorac Cardiovasc Surg. 2001;13:468-75.

18. Athanasuleas C, Stanley A, Dor V, et al. Surgical anterior ventricular restoration (SAVER) in the dilated remodeled ventricle after anterior myocardial infarction. J Am Coll Cardiol. 2001;37:1999-209. 